\title{
Combination of nulling interferometer and modified pupil for observations of exo-planets
}

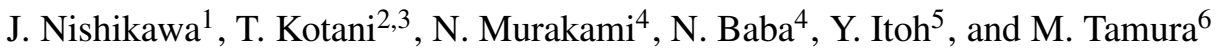 \\ ${ }^{1}$ MIRA project, National Astronomical Observatory of Japan, Osawa, Mitaka, Tokyo 181-8588, Japan \\ e-mail: jun.nishikawa@nao.ac.jp \\ 2 LESIA, Observatoire de Paris, section Meudon, France \\ e-mail: takayuki.kotani@obspm.fr \\ 3 Department of Astronomy, Graduate School of Science, University of Tokyo, Bunkyo-ku, Tokyo 113-0033, Japan \\ ${ }^{4}$ Department of Applied Physics, Graduate School of Engineering, Hokkaido University, Sapporo 060-8628, Japan \\ e-mail: [naoshi; nbaba]@eng.hokudai.ac.jp \\ 5 Graduate School of Science and Technology, Kobe University, Nada, Kobe 657-8501, Japan \\ e-mail: yi toh@kobe-u.ac.jp \\ ${ }^{6}$ Optical and Infrared Division, National Astronomical Observatory of Japan, Mitaka, Tokyo 181-8588, Japan \\ e-mail: hide@subaru.naoj.org
}

Received 26 August 2002 / Accepted 25 November 2004

\begin{abstract}
We consider a combination of two techniques, namely the nulling interferometer and halo suppression by modified pupil such as shaped pupils and apodized square apertures, which achieves very high dynamic range, in order to detect faint extra-solar planets around nearby stars. The effects of the nulling interferometer and the modified pupil are independent and in combination hence multiplied. We show that one can achieve higher dynamic range in the case of a resolved star than with either method alone. By numerical simulations, we show that the combination method can achieve dynamic range levels of $10^{-10}$ at $3 \lambda / D$. Used alone, the two-telescope interferometer would give the nulling depth of $10^{-3}$ whilst the halo suppression by a shaped aperture would do $10^{-7}$, with a point-spread-function core radius less than $3 \lambda / D$ for a shaped aperture. The introduction of the modified pupil has the same effect whether it is made at the entrance apertures of the interferometer or at a re-imaged common pupil plane after the nulling interferometry. From another point of view, a nulling interferometer works as pre-optics in front of any single telescope methods, which reduces the intensity of a resolved source transmitting some uniform wavefront residuals.
\end{abstract}

Key words. instrumentation: high angular resolution - techniques: interferometric - techniques: high angular resolution telescopes - stars: planetary systems

\section{Introduction}

Nulling interferometry (Bracewell 1978) is one of the useful methods of achieving high dynamic range observations especially for the direct detection of extra-solar planets (e.g. Woolf \& Angel 1998). Darwin (Fridlund 2000) and Terrestrial Planet Finder (Beichman et al. 1999) originally have been based on this concept at mid-infrared wavelengths.

Another method of achieving high dynamic range is the advanced coronagraph, which extinguishes the central star light within a single telescope optics. The nulling coronagraph is a particular implementation using an interferometric null for the star by putting a phase mask at the center of the image plane (Roddier \& Roddier 1997; Rouan et al. 2000).

The third method is the modified pupil, which suppresses the halo of the point spread function (PSF) relative to the core intensity. Shaped apertures (Jacquinot \& Roizen-Dossier 1964;
Spergel 2001) and apodized square or rectangular apertures (Nisenson \& Papaliolios 2001) are examples. Various modifications of the method are seen in recent studies (e.g. Kasdin et al. 2003; Guyon 2003). The coronagraph and the modified pupil method are typically used at optical and near infrared wavelengths.

The advanced coronagraph has many variations (Baudoz et al. 2000; Baba et al. 2002; Guyon \& Roddier 2002; Kuchner $\&$ Traub 2002; Soummer et al. 2003), and some of the nulling coronagraph designs can provide total extinction for an on-axis point source (Rouan et al. 2002; Abe et al. 2003). Another kind of nulling coronagraph was also able to achieve this when combined with entrance aperture apodization (Aime et al. 2002; Soummer et al. 2003). But considerable residual light from offaxis rays remains an issue (Guyon et al. 1999; Rouan et al. 2000; Riaud et al. 2003). 
The nulling interferometer is, in principle, able to extinguish an on-axis point source perfectly, even for wide-band light (Bracewell 1978). Several teams are working to achieve high performance with nulling interferometry, for example, wide-band nullers (Shao \& Colavita 1992; Serabyn et al. 1999; Baba et al. 2001; Ollivier et al. 2001) or differential wavelength techniques (Danchi et al. 2003). On the other hand, the twotelescope Bracewell interferometer remains limited by residual light from off-axis rays. In order to reach better null for resolved stars, a nulling interferometer with more than two telescopes and their various configurations were studied (Angel \& Woolf 1997; Mennesson \& Marriotti 1997), but the combination with a nulling coronagraph has not been studied yet, nor the combination with a modified pupil. If one look at a SunEarth-like system at a distance of $5 \mathrm{pc}$, the planet is found at 200 milliarcsec (mas) separation from the central star, which has a 2 mas resolvable apparent diameter, producing significant leakage intensities for the nulling interferometer and also for the nulling coronagraph, which would be a hindrance to the direct detection of the planet.

In the present paper, in order to reduce the off-axis leakage light from resolved stars, we study the combination of the nulling interferometer and the modified pupil method. The combination is effective in those cases where the point-spreadfunction core radius of each element telescope is a few times smaller than the separation angle between a target exo-planet and the central star. For example, $600 \mathrm{~nm}$ observations by an interferometer of two $3 \mathrm{~m}$-apertures which can be extracted as sub-apertures of a single $6 \mathrm{~m}$ telescope, or observations by a telescope with LBT-like parameters (Hinz et al. 2003) at 1.6 microns.

In Sect. 2, the theoretical principle of the combination is described, and its performance is confirmed by a simple numerical simulation in Sect. 3. A nulling interferometer is shown to work as pre-optics reducing the source intensity by a fixed amount, independent of the modified pupil method. It is convenient to put the nulling interferometer in front of any other optics, since it keeps the wavefront flat. A discussions of the performance of the combination and of other possible combinations, for instance with the nulling coronagraph or the modified Lyot stop, are given in Sect. 4.

\section{General description}

A general description of the combination concept is shown below and in Fig. 1. The complex amplitude of the wavefront from a source at the entrance pupil plane of a nulling interferometer can be expressed as

$A(\boldsymbol{x}, \boldsymbol{s})=E(\boldsymbol{s}) \mathrm{e}^{i \phi(\boldsymbol{x}, \boldsymbol{S})}$,

where $\boldsymbol{x}$ is a two-dimensional coordinate system in the pupil plane, and $s$ is the projected component of the unit pointing vector to a source onto this plane. Therefore, $\boldsymbol{s}=\mathbf{0}$ denotes the wavefront from an on-axis unresolved star whose pointing direction is perpendicular to the pupil plane. $E(s)$ is the real

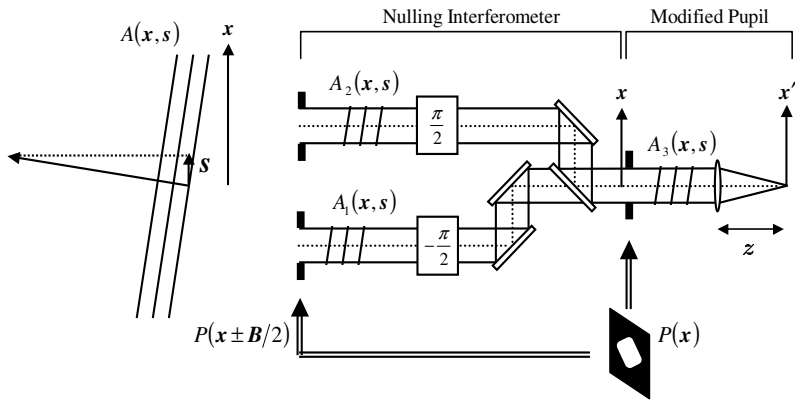

Fig. 1. Description of the proposed combination of the nulling interferometer and the modified pupil method. As described in Sect. 2, the on- or off-axis star light wavefront $A(x, s)$ is extracted by two apertures at $P(\boldsymbol{x} \pm \boldsymbol{B} / 2)$, shown as $A_{1}(\boldsymbol{x}, \boldsymbol{s})$ and $A_{2}(\boldsymbol{x}, \boldsymbol{s})$, and combined into $A_{3}(\boldsymbol{x}, \boldsymbol{s})$ with phase difference $\pi$ at an optical axis where an equivalent pupil is seen as $P(\boldsymbol{x})$.

positive amplitude of the electric field, and the phase $\phi(x, s)$ is given by

$\phi(x, s)=-\frac{2 \pi}{\lambda}(x \cdot s)$.

Here, denotes inner product and $\lambda$ is the wavelength. The wavefronts received by the two telescopes of the nulling interferometer at positions $\boldsymbol{x}=-\boldsymbol{B} / 2$ and $\boldsymbol{x}=+\boldsymbol{B} / 2$ are described by

$A_{1}(\boldsymbol{x}, s)=P(\boldsymbol{x}) A\left(\boldsymbol{x}-\frac{\boldsymbol{B}}{2}, s\right)$

and

$A_{2}(\boldsymbol{x}, s)=P(x) A\left(x+\frac{B}{2}, s\right)$,

respectively, here the $\boldsymbol{x}$ coordinates for $A_{1}$ and $A_{2}$ are considered after the beams are combined. $P(\boldsymbol{x})$ is the pupil function used for both telescopes which is an arbitrary shape and apodization, written as

$P(\boldsymbol{x})=\left\{\begin{array}{ll}T & (0 \leq T \leq 1, \text { inside the pupil) } \\ 0 & \text { (outside the pupil) }\end{array}\right.$.

The wavefronts from the two telescopes are combined with an achromatic phase difference $\pi$ for an on-axis source and interfere destructively. Then, the electric field in the combined pupil plane is given by

$$
\begin{aligned}
A_{3}(\boldsymbol{x}, \boldsymbol{s}) & =A_{1}(\boldsymbol{x}, \boldsymbol{s}) \mathrm{e}^{-i \pi / 2}+A_{2}(\boldsymbol{x}, \boldsymbol{s}) \mathrm{e}^{+i \pi / 2} \\
& =P(\boldsymbol{x}) A(\boldsymbol{x}, \boldsymbol{s}) \epsilon(\boldsymbol{B}, \boldsymbol{s}),
\end{aligned}
$$

where

$\epsilon(\boldsymbol{B}, \boldsymbol{s})=2 \sin \left(\frac{\pi}{\lambda}(\boldsymbol{B} \cdot \boldsymbol{s})\right)$.

Here, we assume that the effect of diffraction due to the propagation of the light beams can be neglected, which is possible by re-imaging the pupil plane. Total extinction occurs when $(\boldsymbol{B} \cdot \boldsymbol{s})=n \lambda(n$ : integer), therefore only when $\boldsymbol{s}=\mathbf{0}$ for white light. Consequently, the output wavefront from the nulling interferometer has an amplitude reduced by $\epsilon$, a function of the 


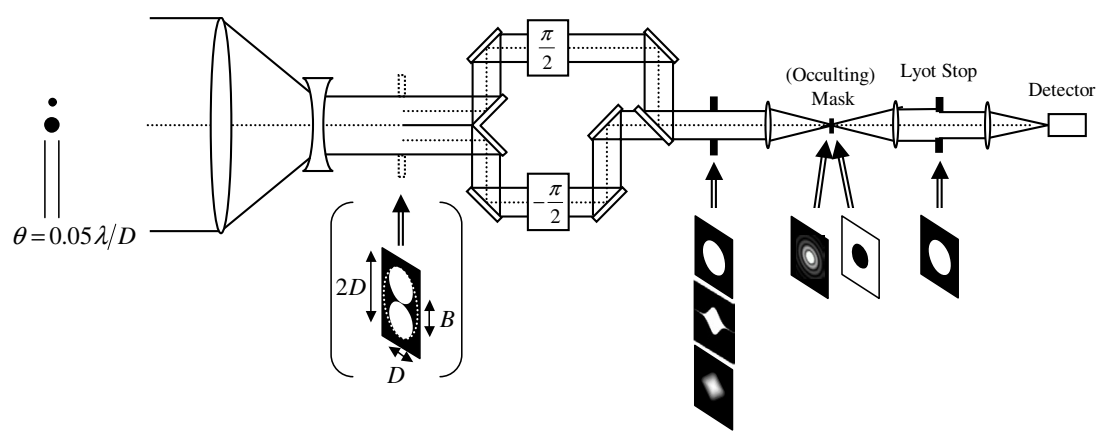

Fig. 2. Schematic of a possible optical implementation of the concept and the simulation parameters. In the collimated beam of a single telescope, two contacted entrance apertures of an equivalent diameter $D$ are extracted. The two beams are combined destructively with a nulling interferometric combiner. Then a modified pupil telescope is applied to produce a high dynamic range image which is followed by an occulting mask hiding the bright core, a collimator, Lyot stop, a focusing lens, and a camera, suitably adjusted to avoid saturation.

baseline $\boldsymbol{B}$ and the source position $\boldsymbol{s}$, with the wavefront remaining flat. Note in Eq. (6) that the modified pupil function operating at the entrance telescopes is identical to that at the corresponding pupil plane after the nulling combiner.

The intensity distribution in the focal plane is given by the squared absolute value of the Fourier transform of $A_{3}(\boldsymbol{x}, \boldsymbol{s})$. By using the notation of the Fourier optics of a scaled transform (Goodman 1996), Eqs. (1) and (2), this can be described as follows.

$$
\begin{aligned}
I\left(x^{\prime}, s^{\prime}\right) & =\left|\hat{A}_{3}\left(x^{\prime}, s^{\prime}\right)\right|^{2} \\
& =\frac{1}{(\lambda z)^{2}}\left|\hat{P}\left(x^{\prime}-s^{\prime}\right)\right|^{2} E^{2}\left(s^{\prime}\right) \epsilon^{2}\left(B, s^{\prime}\right),
\end{aligned}
$$

where $\boldsymbol{x}^{\prime}$ is an image plane two-dimensional coordinate defined as $\boldsymbol{x}^{\prime}=\boldsymbol{x} / \lambda z, \boldsymbol{s}^{\prime}=-\boldsymbol{s} / \lambda, z$ is the focal length of the imaging system, and ${ }^{\wedge}$ denotes the Fourier transform operation. We reuse the notations $E$ and $\epsilon$ after $\boldsymbol{s}$ is transferred to $\boldsymbol{s}^{\prime} .\left|\hat{P}\left(\boldsymbol{x}^{\prime}\right) / \lambda z\right|^{2}$ is the PSF of the modified pupil function $P(\boldsymbol{x})$. Equation (8) shows that the image of a point source with this modified-pupil nulling interferometer is the PSF of the modified pupil function $P(\boldsymbol{x})$ with an intensity of $E^{2}$ reduced by $\epsilon^{2}$, the so-called transmission map, a function of $\boldsymbol{B}$ and $\boldsymbol{s}$ (or $\boldsymbol{s}^{\prime}$ ). Thus the effect of the nulling interferometer and the modified pupil imaging are independent, and multiplied.

From Eq. (8), the final image of an extended source like a resolved star is given by

$$
\begin{aligned}
I\left(x^{\prime}\right) & =\int_{\mathrm{S}} I\left(\boldsymbol{x}^{\prime}, \boldsymbol{s}^{\prime}\right) \mathrm{d} \boldsymbol{s}^{\prime} \\
& =\frac{1}{(\lambda z)^{2}} \int_{\mathrm{S}}\left|\hat{P}\left(\boldsymbol{x}^{\prime}-\boldsymbol{s}^{\prime}\right)\right|^{2} E^{2}\left(\boldsymbol{s}^{\prime}\right) \epsilon^{2}\left(\boldsymbol{B}, \boldsymbol{s}^{\prime}\right) \mathrm{d} \boldsymbol{s}^{\prime} .
\end{aligned}
$$

Here, the integral $\mathrm{S}$ is taken over the surface of the stellar disk. The final image of the resolved star is generated by a convolution of the modified pupil PSF with the nullinginterferometrically dimmed source intensity distribution $E^{2} \epsilon^{2}$. In our simulation results in the next section, the dimming of the shaped-pupil PSF profiles is obvious.

\section{Simulation of a nulling interferometer with modified pupil}

In this section we investigate the performance of the combination of the nulling interferometer and the modified pupils, using numerical simulations.

The simulation parameters that we used for the concept are as can be seen in Fig. 2: we assume a two-telescope interferometer with an entrance pupil of diameter $D$. Setting the baseline $B$ equal to $D$, at an observing wavelength $\lambda=2 \times 10^{-7} D$ we get $\lambda / B=41$ mas. The observed star is a uniform disk of $0.05 \lambda / D$ apparent diameter. This parameter set corresponds to a $3 \mathrm{~m}$ baseline optical interferometer with two $3 \mathrm{~m}$ telescopes observing a Sun-like star at a distance of 5 pc, of 2 mas apparent diameter at a wavelength of $600 \mathrm{~nm}$. A planet at a distance of $1 \mathrm{AU}$, of $<10^{-9}$ contrast at optical wavelengths is seen at 200 mas corresponding to $5 \lambda / D$. We illustrate the case where the two apertures are extracted from a primary mirror, the nulling instruments being more stable than in a separate telescope system. For each point of the star and for each telescope a wavefront electric field is generated on 800-by-800-point twodimensional grids. The wavefront from one of the two telescopes is modified by a phase shift of $\pi$ and summed with the other wavefront, which yields destructive interference, the result of which is pasted on a section of the 8192 by 8192 array used for the pupil. A constructive result with zero phase shift is calculated to normalize the performance of the nulling optics. When we consider the wave front errors, they are added for each aperture wavefront before the summation.

Either a circular or a modified pupil stop is applied to the array. Three kinds of modified pupil are provided here. The boundary curve for the shaped aperture with the Kaiser-Bessel window function (cf. Harris 1978), approximating a prolatespheroidal wave function (cf. Kasdin et al. 2003) is given by

$p(x)=\frac{J_{0}\left(\pi \alpha \sqrt{1-\left(\frac{x}{R}\right)^{2}}\right)}{J_{0}(\pi \alpha)}$,

where $J_{0}$ is the zero-order modified Bessel function of the first kind, $\alpha=2.6, R=400$, and $x(-400 \leq x \leq 400)$ is the pixel number in the pupil array along the axis in the image plane where the intensity profile has the highest contrast. The pupil 
function $P(x)$ is unity when the pixel is entirely inside the area $|y| \leq p(x)$, zero when outside it, and the appropriate fraction for boundary pixels. Here $y$ is an axis perpendicular to $x$ in the array.

Another boundary curve for a sonine-windowed Gaussian aperture is given by

$p(x)=R \mathrm{e}^{-(1.5 x / R)^{2}}\left(1-\left(\frac{x}{R}\right)^{2}\right)^{6}$,

and the pupil function is defined as previously.

Finally, we evaluate a square aperture whose diagonal length is 800 pixels, rotated by 45 degrees and with apodization function within the square pupil (cf. Nisenson \& Papaliolios 2001) for $|x|+|y| \leq 400$ given by

$T(x, y)=\left(1-\left(\frac{x+y}{R}\right)^{2}\right)^{3}\left(1-\left(\frac{x-y}{R}\right)^{2}\right)^{3}$.

FFT is used to calculate the intensity distribution in the image plane. The star diameter is sampled on a 100 pixels square grid and the image profiles corresponding to each pixel are summed.

In Fig. 3a we compare the intensity profiles for the nulling interferometer combined with modified pupils to those with circular pupils. In this case both constructive and destructive conditions are represented. At a distance of $5 \lambda / D$, the effect of the modified pupil is a halo suppression of about $10^{-7}$, for each of the three cases given by Eqs. (10)-(12) while the interferometer nulling depth is $1.4 \times 10^{-3}$, yielding a combined dynamic range enhancement of about $1 \times 10^{-10}$. In this case the image core radius along the high-contrast axis for the Kaiser-Bessel window shaped aperture of Eq. (10) could become less than $3 \lambda / D$. When $\alpha=4$ for the aperture, the dynamic range reaches $1 \times 10^{-13}$ at $5 \lambda / D$ combined with the nulling interferometer, while the image core radius becomes about $4 \lambda / D$.

In Fig. 3b, the same profiles are shown but this time including 0.01 wavelength rms normally distributed wavefront errors. At a distance of $>5 \lambda / D$, the effects of the modified pupils and the nulling interferometer are wavefront error noise limited at $1 \times 10^{-8}$, while the combination method proves effective for $<3 \lambda / D$. The halo noise is found as the background in the case of constructive interference. A noise level of $1 \times 10^{-10}$ is obtained for 0.001 wavelength rms errors. The combination of a nulling interferometer and a modified pupil does not reduce the wavefront error noise.

\section{Discussion}

The nulling interferometer can achieve total extinction for an on-axis source, but off-axis residual intensity is observed depending on the transmission map of the interferometer. The combination of the nulling interferometer and the modified pupil is shown to be more effective for resolved stars in terms of off-axis light reduction than either method by itself, because the effects of the two methods are multiplied. It is possible to adopt here a multiple-aperture nulling interferometer with a better null for a resolved star than that achieved with the Bracewell two-aperture design, for example one with four $3 \mathrm{~m}$ sub-apertures extracted from an $8 \mathrm{~m}$ telescope. When the
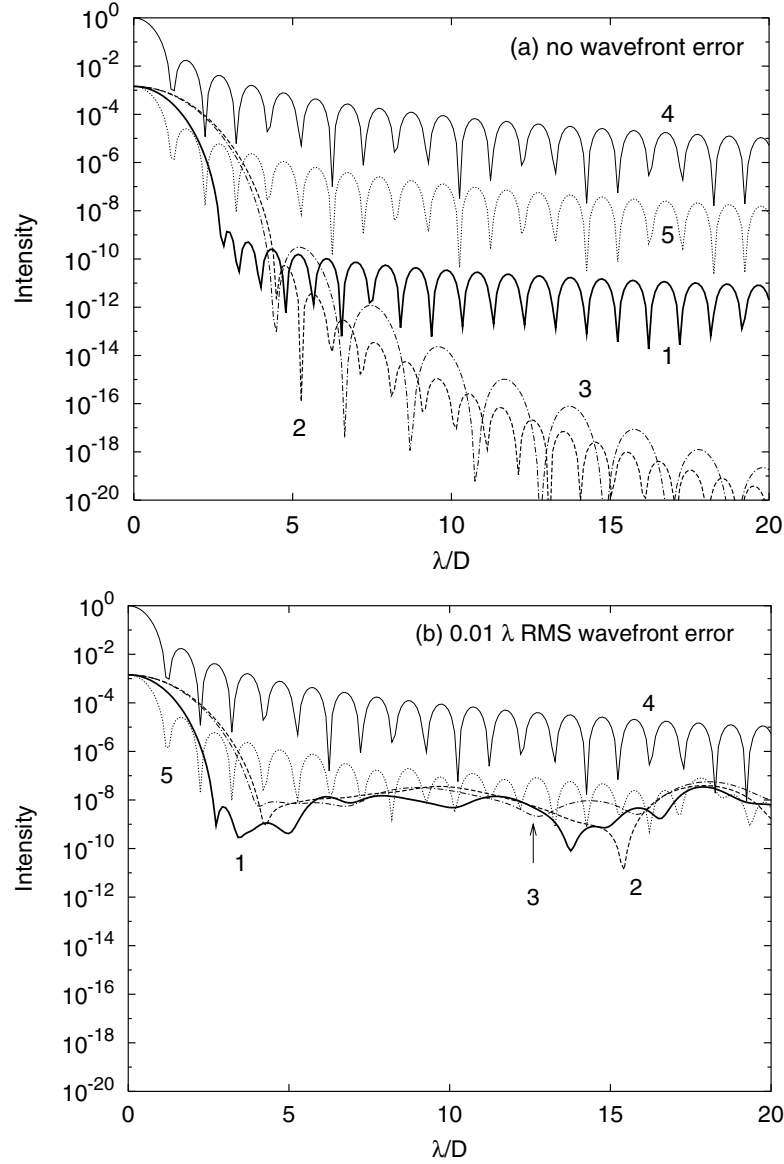

Fig. 3. Intensity profiles along the high-contrast axis for combinations of nulling interferometer with modified pupils. The profile for the Kaiser-Bessel window shaped pupil of Eq. (10) under nulling interferometer destructive conditions is shown (1: bold line), the sonine windowed Gaussian of Eq. (11) by the dashed line 2, and the apodized square of Eq. (12) by the dot-dashed line 3. In the case of the circular aperture we show both constructive conditions (4: solid line) and destructive nulling conditions (5: dotted line). a) is the ideal case of zero wavefront errors, and b) includes normally distributed $0.01 \lambda \mathrm{rms}$ wavefront errors.

nulling performance of the interferometer is $1 \times 10^{-3}$ for a resolved star, the modified pupil telescope is only required to suppress the halo intensity at $1 \times 10^{-7}$ to get a combined intensity of $1 \times 10^{-10}$ at the position of the planet. The low dynamic range requirement for the modified pupil method would favor a design with higher performance, such as a small PSF core radius and a high throughput. Various modified pupil telescope designs (e.g. Kasdin et al. 2003; Guyon 2003) are available for the combination.

Equation (6) shows that the modification of the two entrance pupils of the nulling interferometer is equivalent to that of a corresponding pupil plane after the nulling beam combiner. Therefore the front nulling interferometer is considered to be independent pre-optics of a back-side modified pupil single telescope, which simply reduces the source intensity according to the transmission map. Note that the planet intensity is also affected by the map and rotation of the interferometer is required to get a sensitivity for the whole field of view (Bracewell 1978). The upstream nulling interferometer 
can be conveniently put in front of any other optics without wavefront change. This implies that an additional nulling operation is applicable when the wavefront electric field in the output pupil plane has a non-random and simple distribution after any previous nulling interferometric or coronagraphic operation. Consequently the nulling coronagraph method for a single telescope optics, instead of the modified pupil method, is considered to be effective as the back optics of the nulling interferometer.

In Fig. 2, a schematic of a possible optical layout for the concept with a camera optics is shown. In the collimated beam of a single telescope, after the nulling interferometer, a modified pupil telescope is placed to produce a high dynamic range image, which is followed by an occulting mask to hide the bright core, a collimator, Lyot stop, a focusing lens, and a camera suitably adjusted to avoid saturation. The only stop actually needed is the one at the common location of the modified pupil telescope, i.e. after the combiner.

The nulling coronagraph method has various designs using a simple circular (elliptical) or square (rectangular) pupil, an image plane (phase) mask, and a Lyot stop. Some nulling coronagraph methods without pupil modification can achieve the complete extinction of an on-axis point source (Rouan et al. 2002; Abe et al. 2003), and some designs work when combined with apodized entrance apertures (Aime et al. 2002; Soummer et al. 2003). For any nulling coronagraph, however, as well as for the nulling interferometer, an off-axis source remains un-nulled. The final image intensities including tip-tilt errors were estimated for some coronagraphs (Guyon et al. 1999; Rouan et al. 2000). It was not estimated whether the residual intensity of a resolved star was below $1 \times 10^{-9}$. The rejection factor of stellar light within a radius of $0.012 \lambda / D$ for a four-quadrant phase-mask coronagraph is shown to be limited to 16100 (Riaud et al. 2003). In any case, the residual offaxis light for the nulling coronagraph can be suppressed by the upstream nulling interferometer, yielding a combination concept of the nulling interferometer and the nulling coronagraph methods with/without modified entrance pupil. In Fig. 2, when the nulling coronagraph is used after the nulling interferometer, only the occulting mask should be replaced by a suitable phase mask, with/without modification of the common pupil.

Though an apodized Lyot stop showed halo intensity suppression for the classical coronagraph method (Itoh et al. 1998), all of the apodization has been done at the entrance pupil for the nulling coronagraphy, achieving total extinction for an onaxis point source (Aime et al. 2002; Soummer et al. 2003). However, it is not proved that the modified Lyot stop is ineffective especially for the case of a resolved star. When there is a significant residual amplitude of partially-extinct off-axis regular light from a resolved star surface, if the residual wavefront has a large uniform component, the additional application of a modified Lyot stop might suppress the halo intensity in the final image plane. Eventual off-axis light from a star in the presence of tip-tilt errors would be in a similar situation. Some combinations of the nulling interferometer, the modified entrance pupil, the advanced coronagraph, and the modified Lyot stop might have the potential to achieve a higher dynamic range for realistic resolved star observations than that obtained by presently known methods. Again looking at Fig. 2 in this case, the pupil, the occulting mask, and the Lyot stop would be respectively replaced by a modified pupil, an image plane (phase) mask, and modified Lyot stop by using a variation of the modified pupil telescopes (e.g. Kasdin et al. 2003; Guyon 2003). The study of a combination of the nulling coronagraph with a modified Lyot stop in the case of a resolved star will be published in a future paper (Murakami et al. in preparation).

Achromatic interfero-coronagraphy (AIC) is another kind of interferometric nulling coronagraph (Baudoz et al. 2000), which might be used as back optics of the nulling interferometer. Because the AIC output wave after recombination is similar to that of a nulling interferometer, it might be used as pre-optics of another nulling coronagraph, though it produces axi-symmetric duplicated images.

As seen in Fig. 3, wavefront errors limit the halo suppression level, therefore the nulling interferometer and the modified pupil method are only effective with sufficiently high-quality optics. In the telescope optics, there are many parts producing wave front errors and scattered light: mirror surfaces, apodization filters, phase shifters, beam splitters, phase masks, dust, and so on. A theoretically high performance method might become impossible experimentally because of these difficulties. The use of polarization characteristics (e.g. Baba \& Murakami 2003) or differential wavelength techniques (e.g. Danchi et al. 2003) is one of the candidates to develop high dynamic range optics insensitive to such disturbances. To search realistically possible high performance nulling setup for resolved star observations especially in optical coronagraphs requiring very high dynamic range is as important as theoretical and numerical studies.

\section{Conclusions}

We have presented a combination of a nulling interferometer in front of a modified pupil telescope as a candidate for the high dynamic range optics for direct observation of exo-planets. The nulling interferometer can produce the total extinction for an on-axis point source but it transmits leakage light for a resolved star with off-axis rays. With the combination presented here, the effects of the nulling interferometer and the modified pupil are independent and they are multiplied, resulting in higher dynamic range than either method by itself. For the source intensity reduction effect of the nulling interferometer (e.g. $10^{-3}$ ) in the combination methods to reach the desired total dynamic range of $10^{-10}$, the modified pupil is required to provide a lower contrast halo suppression (e.g. $10^{-7}$ ) which is a welcome situation for helping exo-planet-finding telescopes to reduce the modified-pupil PSF core radius (e.g. $\leq 3 \lambda / D)$ and to increase throughput. The present combination is useful for the case where the point-spread-function core radius of each element telescope is a few times smaller than the separation angle of the target exo-planet from the central star. An example of the condition is a $600 \mathrm{~nm}$ wavelength observation of a SunEarth-like system at a distance of $5 \mathrm{pc}$, with an apparent stellar diameter of 2 mas and a separation between the star and the planet of 200 mas, using a $3 \mathrm{~m}$ baseline interferometer which is composed of two $3 \mathrm{~m}$ sub-apertures with a diffraction limit 
of 41 mas extracted from a $6 \mathrm{~m}$ telescope. The introduction of a modified pupil has the same effect whether it is made at the entrance aperture of the interferometer or at a re-imaged common pupil plane after the nulling interferometry. From another point of view, a nulling interferometer is shown to work as preoptics which reduces the source intensity in front of any single telescope optics, just like the modified pupil method. An additional nulling operation is applicable when the wavefront at the output pupil plane for each point of the resolved source has a uniform distribution after any previous nulling operation. Then, after the nulling interferometer, instead of the modified pupil, the nulling coronagraph method for a single telescope optics is considered to be effective for extinguishing off-axis light, and further operations yielding a high dynamic range would be possible if there remained some uniform wavefront residuals after the two nulling methods. In analogy with the present concept, it is important to investigate various optical designs and combinations which have not yet been explored but have the potential to achieve higher dynamic range than that obtained by presently known methods and combinations. We are interested in making modifications to known methods including the proposals of the present paper in order to achieve high dynamic range with available optical components.

Acknowledgements. The authors acknowledge Prof. M. Yoshizawa, members of Mitaka optical and InfraRed Array (MIRA) project, and members of Japanese TPF working group for stimulating discussions and their constant encouragement with this work. We are grateful to Dr. C. Aime, the referee, for helpful advice on improving the manuscript. Y.I. is supported by a Grant-in-Aid for Scientific Research No. 16740256 of the MEXT.

\section{References}

Abe, L., Domiciano de Souza, A., Jr., Vakili, F., \& Gay, J. 2003, A\&A, 400,385

Aime, C., Soummer, R., \& Ferrari, A. 2002, A\&A, 389, 334

Angel, J. R. P., \& Woolf, N. J. 1997, ApJ, 475, 373

Baba, N., Murakami, N., \& Ishigaki, T. 2001, Opt. Lett., 26, 1167
Baba, N., Murakami, N., Ishigaki, T., \& Hashimoto, N. 2002, Opt. Lett., 27, 1373

Baba, N., \& Murakami, N. 2003, PASP, 115, 1363

Baudoz, P., Rabbia, Y., \& Gay, J. 2000, A\&AS, 141, 319

Beichman, C. A., Woolf, N. J., \& Lindensmith, C. A. 1999, The Terrestrial Planet Finder, JPL Publication 99-3 (Pasadena: JPL)

Bracewell, R. N. 1978, Nature, 274, 780

Danchi, W. C., Deming, D., Kuchner, M. J., \& Seager, S. 2003, ApJ, 597, L57

Fridlund, M. 2000, DARWIN, The Infrared Space Interferometer, The Search for Terrestrial Exoplanets and High Resolution Imaging of the Universe, ESA SCI-12 (Noordwijk: ESA)

Goodman, J. W. 1996, Introduction to Fourier Optics, 2nd ed. (McGraw-Hill)

Guyon, O. 2003, A\&A 404, 379

Guyon, O., \& Roddier, F. 2002, A\&A 391, 379

Guyon, O., Roddier, C., Graves, J. E., et al. 1999, PASP, 111, 1321

Harris, F.J. 1978, Proc. IEEE, 66, 51

Hinz, P. M., Angel, J. R. P., McCarthy, D. W., Jr., Hoffman, W. F., \& Peng, C. Y. 2003, Proc. SPIE, 4838, 108

Itoh, Y., Takato, N., Takami, H., \& Tamura, M. 1998, PASJ, 50, 55

Jacquinot, P., \& Roizen-Dossier, M. B. 1964, Progress in Optics, 3 , 29

Kasdin, J. N., Vanderbei, R. J., Spergel, D. N., \& Littman, M. G. 2003, ApJ, 582, 1147

Kuchner, M. J., \& Traub, W. A. 2002, ApJ, 570, 900

Mennesson, B., \& Mariotti, J. M. 1997, Icarus, 128, 202

Nisenson, P., \& Papaliolios, C. 2001, ApJ, 548, L201

Ollivier, M., Mariotti, J. -M., Léger, A., et al. 2001, A\&A 370, 1128

Riaud, P., Boccaletti, A., Baudrand, J., \& Rouan, D. 2003, PASP, 115, 712

Roddier, F., \& Roddier, C. 1997, PASP, 109, 815

Rouan, D., Riaud, P., Boccaletti, A., Clenet, Y., \& Labeyrie, A. 2000, PASP, 112, 1479

Rouan, D., Riaud, P., \& Baudrand, J. 2002, in Beyond Conventional Adaptive Optics, ed. E. Vernet, R. Ragazzoni, S. Esposito, \& N. Hubin (Garching ESO), Proc. ESO Conf. 58, 193

Shao, M., \& Colavita, M. 1992, ARA\&A, 30, 457

Serabyn, E., Wallace, J. K., Hardy, G. J., Schmidtlin, E. G. H., \& Nguyen, H. T. 1999, Appl. Opt. 38, 7128

Soummer, R., Aime, C., \& Falloon, E. P. 2003, A\&A, 397, 1116

Soummer, R., Dohlen, K., \& Aime, C. 2003, A\&A, 403, 369

Spergel, D. N. 2001, preprint [arXiv: astro-ph/0101142]

Woolf, N. J., \& Angel, J. R. P. 1998, ARA\&A, 36, 507 